\title{
Sea State Characterization Using Experimental One-Dimensional Radar Signatures and Fractal Techniques
}

\author{
Georgios Pouraimis, Apostolos Kotopoulis, Basil Massinas, Panayiotis Frangos* \\ School of Electrical and Computing Engineering, National Technical University of Athens, \\ 9, Iroon Polytechniou Str., 15773 Zografou, Athens, Greece \\ pfrangos@central.ntua.gr
}

\begin{abstract}
This paper presents a novel method of sea state characterization by using four criteria, which are applied to normalized experimental Synthetic Aperture Radar (SAR) one-dimensional signatures (range profiles), provided to our research group by SET 215 Working Group on "SAR radar techniques". These criteria are the "Fractal Dimension", "Fractal Length", "Variance $\sigma^{2}$ ", and "Power Spectrum Density - Least Squares". The "Fractal Dimension" and "Fractal Length" criteria, which appear to be the most important out of the four criteria, use the "blanket" technique to provide sea state characterization from SAR radar range profiles. It is based on the calculation of the area of a "blanket", corresponding to the range profile under examination, and then on the calculation of the corresponding "Fractal Dimension" and "Fractal Length" of the range profile. The main idea concerning this proposed technique is the fact that normalized SAR radar range profiles, corresponding to different sea states, produce different values of "Fractal Dimension" and "Fractal Length" for all angles of incidence examined here. As a result, a sea state characterization technique for two different sea states (turbulent and calm sea) is presented in this paper.
\end{abstract}

Index Terms-Backscattering of electromagnetic waves from rough surfaces; Fractal dimension; One-dimensional radar range profiles; Sea state characterization; Synthetic Aperture Radar (SAR).

\section{INTRODUCTION}

Fractals can describe an unlimited number of complex patterns that resemble in different scales and are used as a mathematical tool for a variety of applications, such as image analysis and sorting, applied electromagnetism, etc. [1]-[7]. The indistinguishable structure on different scales is a basic feature of fractals. Accordingly, fractals can illustrate a certain very strong form of geometric complexity across multiple data sets, as well as SAR images. Synthetic Aperture Radar (SAR) images can be considered as fractals for a certain range of magnification. In addition, fractal objects have unique properties and features that may be related to their geometric structure.

Previous research in the area of sea clutter investigations by using radar techniques and fractal mathematics methods can be found in [8]-[23]. As opposed to those references, in

Manuscript received 30 October, 2020; accepted 21 March, 2021.

This research was supported in part under the "International Mobility Programme" by National Technical University of Athens (NTUA)/EU. the present paper, the main objective is to examine the sea state characterization problem by using real SAR backscattered data and fractal techniques (for the latter see, e.g., [23]-[31]). Then, in this paper of ours, we use four different criteria: the 'Fractal Dimension', the "Fractal Length", the "Variance $\sigma^{2}$ ", and the "Power Spectral Density - Least Squares". The first two criteria are considered here the main ones, following the method by Peleg et al. [23], which has also been applied in the past to real Synthetic Aperture Radar (SAR) images, using the "blanket" technique, to provide useful information about SAR image classification, as reported by Malamou et al. [24].

This paper uses the recorded sea clutter radar data, which were collected during the "NEMO 2014" trials in Taranto, Italy, using FFI (i.e., "Norwegian Institute of Defense", Oslo, Norway) PicoSAR X-band radar as input to a specific SET Working Group. The experiment took place in the Taranto bay in southern Italy on 23 and 24 September 2014. The first day the weather was quite windy, thus creating a rather turbulent sea, in comparison with the second day, during which the sea surface was almost calm.

\section{EXPERIMENTAL RADAR DATA}

The geometry of the sea state characterization problem is shown in Fig. 1. Here, a helicopter (with PicoSAR radar inside) rises vertically, whereas maintaining its steady position (latitude and longitude), and transmits electromagnetic (EM) radar pulses towards the sea. In addition, it records the azimuth angle with high sampling density in the grazing angle.

During the experiment performed by FFI in September 2014 (NEMO trials), the helicopter kept low vertical velocity and negligible horizontal velocity (helicopter movement from down to up). The first day (23/9/2014), the wind speed was reported in the range from $10 \mathrm{~m} / \mathrm{s}$ to $12 \mathrm{~m} / \mathrm{s}$ (rather high wind speed) and the helicopter pilot kept the direction of the antenna beam up-wind (i.e., the direction of radar pulses - EM wave propagation in the opposite direction of the wind speed) within a $20^{\circ}$ window in the horizontal (azimuthal) direction, as grazing angles $\theta_{g}$ (see Fig. 1) scanned from $3^{\circ}$ to $37^{\circ}$. The time of the full grazing angle span was approximately 4 minutes.

During the second day (24/9/2014), the wind speed was 
very low ( $1 \mathrm{~m} / \mathrm{s}-2 \mathrm{~m} / \mathrm{s}$, which sometimes died out locally) and the range of grazing angles was from $4^{\circ}$ to $38^{\circ}$ with a slight drift in the azimuth pointing angle of the bore sight of no more than $20^{\circ}$.

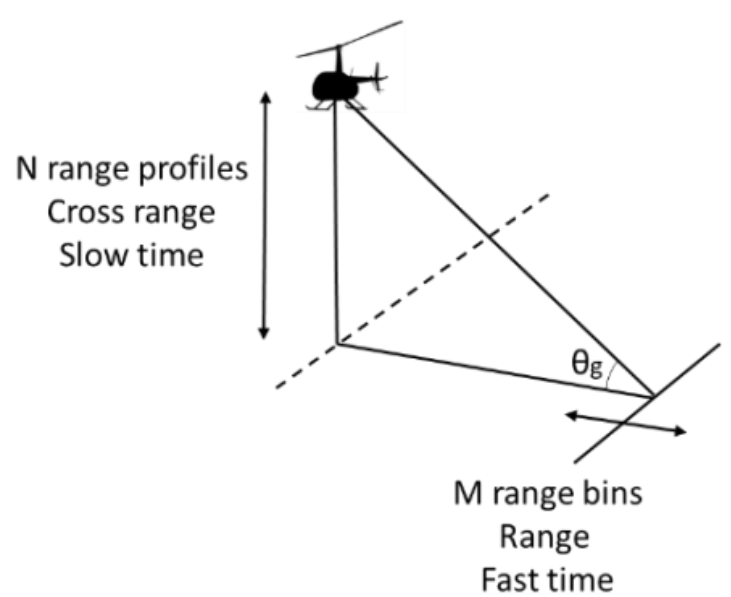

Fig. 1. Geometry of the sea state characterization problem, where the helicopter rises vertically transmitting PicoSAR radar electromagnetic (EM) pulses towards the sea.

However, several practical questions arise about the characterization of a signal that embeds noise. To deal with the presence of noise in the signal, a method is presented here, which initially calculates the average of the range profiles (i.e., "range profile averaging"). For avoiding possible noise spikes in the signal, the number of $N$ samples of the range profiles was set to be equal to 65 , ensuring that this was sufficient to give the most accurate results.

Then the average of the distance profiles was normalized on a scale from 0 to 1 (i.e., "normalized range profiles") and the generated backscattered signal was transformed into the frequency domain (see Fig. 2 and Fig. 3 below).

Observing Fig. 2, the first day (turbulent sea), the range profiles take values from about 0.3 to 1 , whereas during the second day (calm sea), the range profiles take values from 0.6 to 1 (in this case), i.e., smaller variance during the second day. Furthermore, in Fig. 3, the backscattered waves in the frequency domain (i.e., "spectral power") are shown.

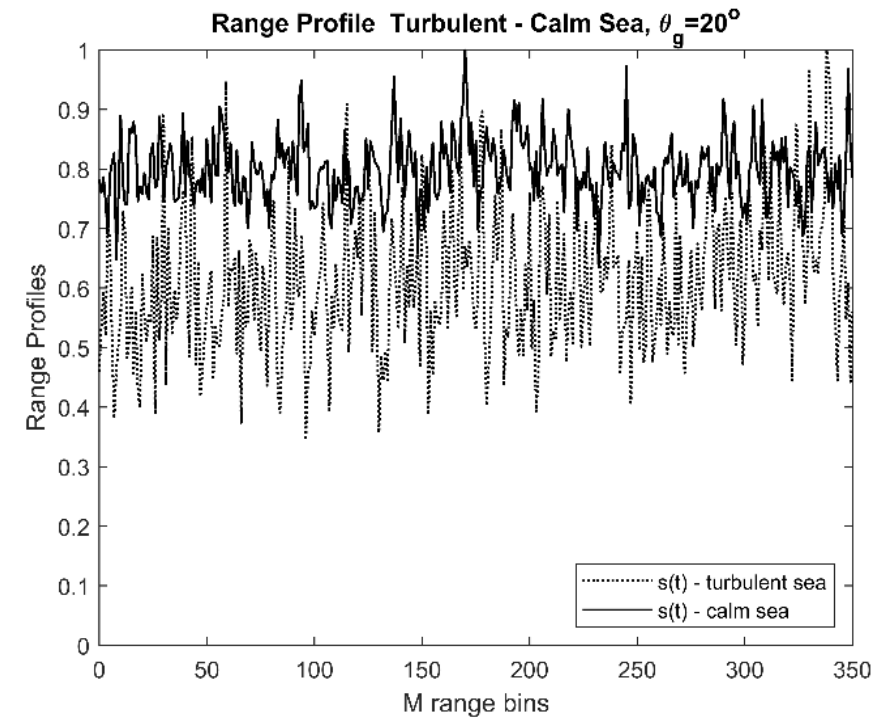

Fig. 2. PicoSAR range profiles for grazing angle $\theta g=20^{\circ}$ for turbulent and calm sea surface in time domain.

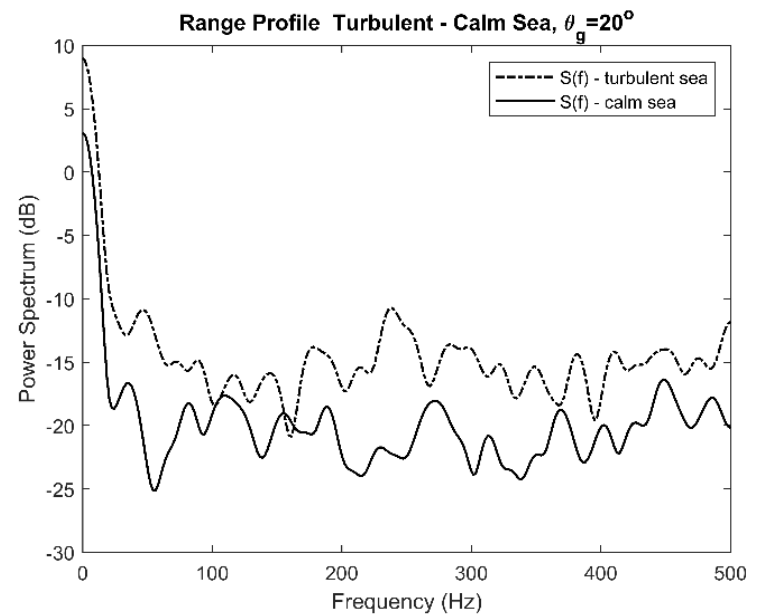

Fig. 3. PicoSAR range profiles for grazing angle $\theta g=20^{\circ}$ for turbulent and calm sea in the frequency domain.

\section{Sea State Characterization Using Two Main FRACTAL CRITERIA AND TWO VERIFICATION CRITERIA}

The sea state characterization criteria that are described in this paper are the two main criteria "Fractal Dimension" and "Fractal Length" and the other two verification criteria, which are the "Variance $\sigma^{2}$ " and "Power Spectral Density Least Squares".

The "Fractal Dimension" and the "Fractal Length" criteria are the first two main methods, which are used for sea state characterization, which calculate the fractal dimension and the logarithmic fractal length of the normalized averaged range profiles in the frequency domain, respectively, for turbulent and calm sea and for grazing angles from $3^{\circ}$ to $37^{\circ}$

The above two criteria use the fractal theory and both fractal criteria use the blanket method, which was introduced by Peleg, Naor, Hartley, and Avnir [25] and was used to characterize the texture of surfaces.

The other two criteria mentioned above (i.e., "Variance $\sigma^{2}$ " and "Power Spectral Density - Least Squares") are used for verification purposes and they are applied directly to the normalized averaged range profiles in the frequency domain.

\section{A. Fractal Dimension and Fractal Length Criteria}

As mentioned above, the blanket method [3] is used here for the calculation of the fractal length and the fractal dimension of the range profiles in the frequency domain. Initially, in the paper by Peleg, Naor, Hartley, and Avnir [23], the surfaces are classified based on the change of their properties in terms of the change of image resolution. Subsequently, Malamou et al. [24] and Tang, Ma, Xi, Mao, and Suen [25] used the blanket method to characterize SAR images and document images, respectively.

The blanket method was proposed [23] to measure the area of irregular surfaces, which had been studied earlier by Mandelbrot [3]-[5]. The blanket method in one dimension, as applied in this paper, considers that all points, which have a distance $\delta$ on both sides of a range profile, create an area of width $2 \delta$, which is called "strip", defined by an upper and a lower blanket. The functions of the "upper" and "lower" curves of the range profile are provided by the following equations [23], [25]: 


$$
\begin{gathered}
u_{\delta}(i)=\max \left\{u_{\delta-1}(i)+1, \max _{|m-i| \leq 1} u_{\delta-1}(m)\right\} \\
b_{\delta}(i)=\min \left\{b_{\delta-1}(i)-1, \min _{|m-i| \leq 1} b_{\delta-1}(m)\right\}
\end{gathered}
$$

where, in the above iteration scheme, $\delta=0$ corresponds to the initial radar range profile. The area $A_{\delta}$ between the upper and lower curves is calculated using the equations of $u_{\delta}$ and $b_{\delta}$ and is given by the following equation

$$
A_{\delta}=\sum_{i}\left(u_{\delta}(i)-b_{\delta}(i)\right)
$$

Subsequently, the fractal length $\mathrm{L}_{\delta}$ of the curve can be calculated approximately as the ratio of the blanket strip area divided by $2 \delta$, as given by the following equation [23], [25]

$$
L_{\delta}=\frac{\mathrm{A}_{\delta}}{2 \delta}
$$

where, in Fig. 4 and Fig. 5, it can be proved [23], [25] that $2 \delta$ is approximately the vertical distance between the "upper blanket curve" and the "lower blanket curve".

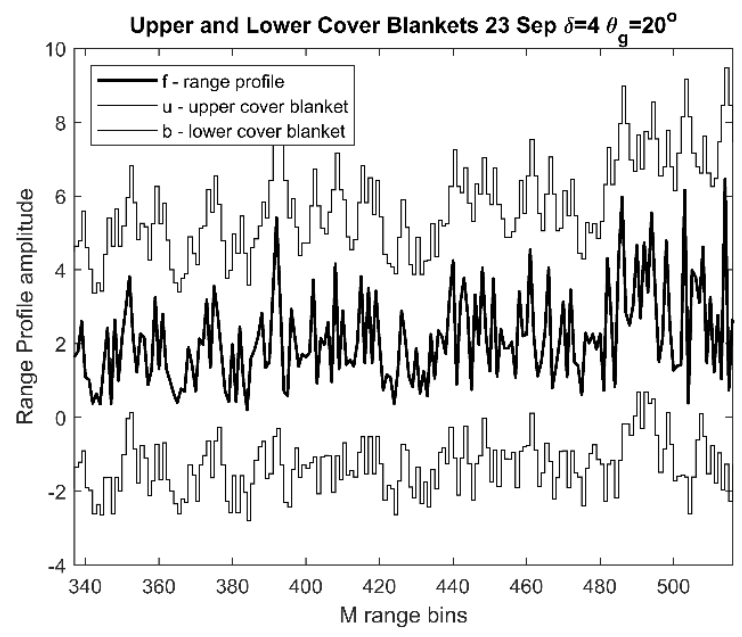

Fig. 4. One-dimensional range profile and upper and lower blanket curves for turbulent sea $(\delta=4)$.

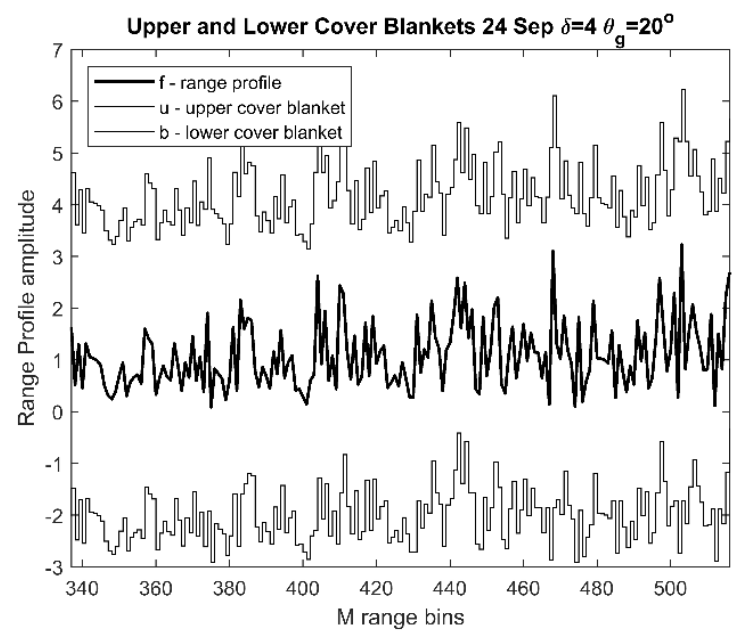

Fig. 5. One-dimensional range profile and upper and lower blanket curves for calm sea $(\delta=4)$.

Equivalently, it can be proved [23], [25] that the fractal length $\mathrm{L}_{\delta}$ can also be calculated through the following formula

$$
L_{\delta}=\frac{\left(\mathrm{A}_{\delta}-\mathrm{A}_{\delta-1}\right)}{2} .
$$

Finally, the fractal dimension $F D_{\delta}$ of the normalized averaged range profile is calculated by [23], [25]

$$
F D_{\delta}=1-\frac{\left(\log A_{\delta}-\log A_{\delta-1}\right)}{(\log \delta-\log (\delta-1))} .
$$

Note that in the iteration procedure described in (1)-(6) above, $\delta=1$ corresponds to the "full resolution" of the range profile, while increasing values of $\delta$ correspond to poorer and poorer resolution of the range profile $(\delta=2,3,4, \ldots)$.

As an example of the above method of calculations for the actual radar data considered in this paper, Fig. 4 and Fig. 5 show the upper and lower curves of the "blanket method" for the radar range profiles, for $\delta=4$ iterations and for grazing angle $\theta_{g}=20^{\circ}$.

\section{B. Variance $\sigma^{2}$ Criterion}

The calculation of the "variance $\sigma^{2}$ " of range profiles in the frequency domain is also used here as a supplementary criterion for sea state characterization for all grazing angles from $5^{\circ}$ to $35^{\circ}$. The variance $\sigma^{2}$ is given by

$$
\sigma^{2}=\frac{1}{f_{\max }} \int_{f=0}^{f_{\max }}\left(S(f)-S_{m}\right)^{2} d f
$$

where $S_{m}$ represents the average value of $S(f)$.

\section{Power Spectrum Density and Least Squares Approximation Criterion}

The power least squares approximation criterion applies to the power spectrum of the normalized average signal in the frequency domain and calculates the slope of the line resulting from the approximation of the least squares to the power spectrum signal [8], [9].

This criterion is used to verify the validity of the two main criteria based on the fractal theory mentioned above. A similar study has been performed by Lo, Leung, Litva, and Haykin [8] and $\mathrm{Hu}$, Tung, and Gao [9], but no similar criterion has been used to date to compare and characterize different rough surfaces.

Using the power spectrum density, the line of the least squares of first degree is calculated, which means that the line is of the form $\alpha x+\beta$. This line can be calculated by the whole set of values of the signal power density for turbulent and calm sea, but for reasons of simplicity, this line is calculated here only for the first ten lower frequencies since the power at low frequencies appears to have higher values and actually, under these circumstances, we are interested here in the slope of this line itself.

\section{Sea State Characterization Results Using the FOUR PROPOSED CRITERIA}

\section{A. Fractal Dimension Criterion Results}

The "Fractal Dimension" criterion calculates the fractal dimension of the normalized average signal, where averaging takes place for $N=65$ range profiles (for noise reduction purposes, as explained above), and ultimately by 
using the blanket method in the frequency domain, as this method was briefly described above.

The results of the simulations comparing the fractal dimension for turbulent and calm sea for grazing angles $10^{\circ}$ and $25^{\circ}$ are shown in Fig. 6 and Fig. 7, respectively.

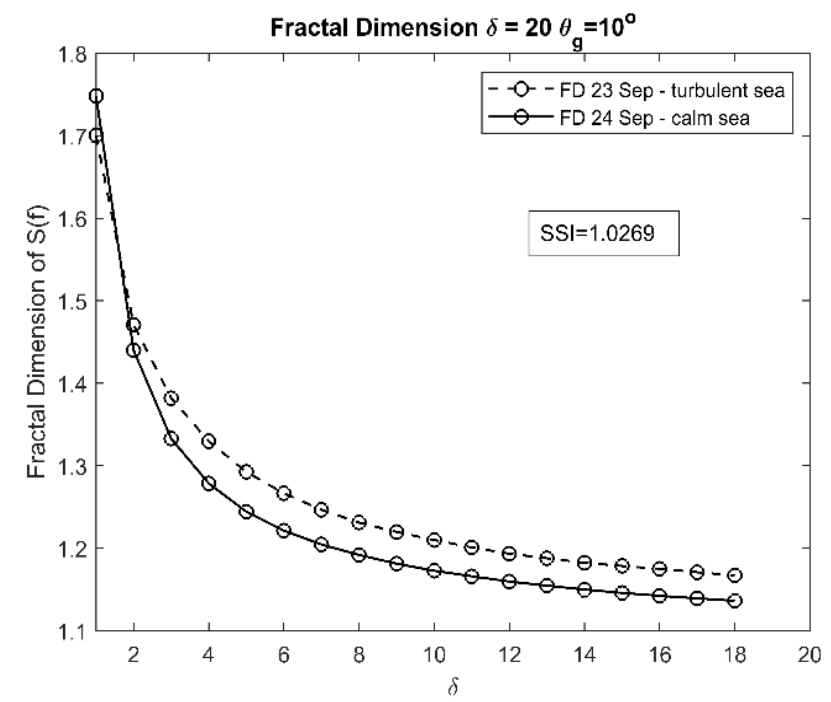

Fig. 6. Fractal dimension as a function of iteration $\delta$ for grazing angle $10^{\circ}$.

The results of Fig. 6 and Fig. 7 show that the values of the fractal dimension of the normalized average radar signals (range profiles) during turbulent sea are significantly higher than the corresponding values at calm sea for all values of iteration $\delta$ and for all values of grazing angle $\theta_{g}$ (see also Table I, and discussion of it, below).

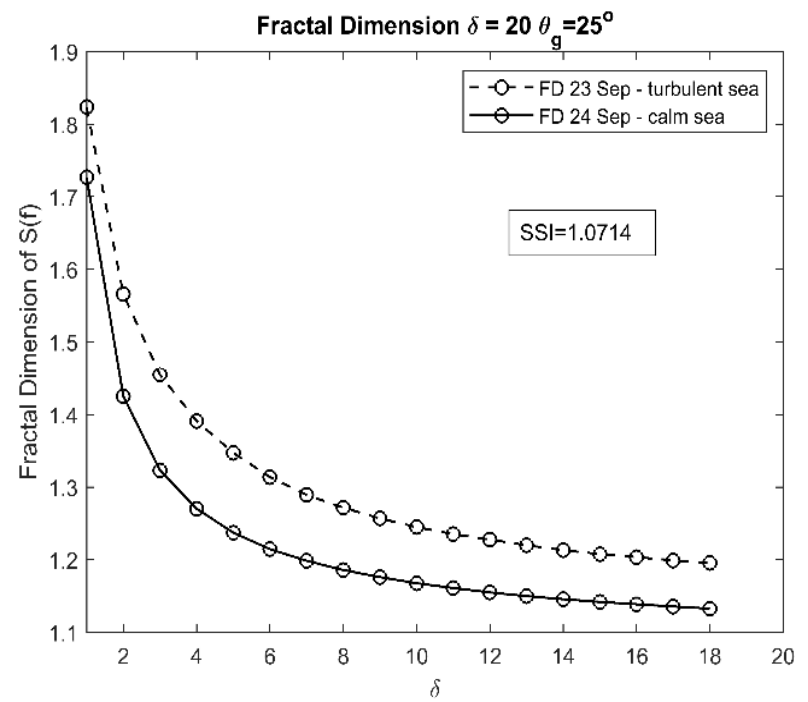

Fig. 7. Fractal dimension as a function of iteration $\delta$ for grazing angle $25^{\circ}$.

Observing the results of the fractal dimension for grazing angles $\theta_{g}$ from $3^{\circ}$ to $37^{\circ}$, it is concluded that the fractal dimension criterion can be used with confidence in the range of grazing angles, despite the presence of electronic noise at the radar receiver.

Finally, the Sea State Index (SSI) is calculated for the fractal dimension criterion. The Sea State Index (SSI) of the fractal dimension criterion is defined here as the ratio of the mean fractal dimension for turbulent sea to the mean fractal dimension for the calm sea in the frequency domain.

TABLE I. SEA STATE INDEX (SSI) FOR FRACTAL DIMENSION.
\begin{tabular}{|c|c|}
\hline Angle & FSI \\
\hline $5^{\circ}$ & 1.043 \\
\hline $10^{\circ}$ & 1.026 \\
\hline $15^{\circ}$ & 1.206 \\
\hline $20^{\circ}$ & 1.063 \\
\hline $25^{\circ}$ & 1.071 \\
\hline $30^{\circ}$ & 1.062 \\
\hline $35^{\circ}$ & 1.089 \\
\hline
\end{tabular}

\section{B. Fractal Length Criterion Results}

The second criterion for sea state characterization is performed by examining the logarithmic fractal length. The "Fractal Length" criterion calculates the logarithm of the fractal length of the normalized average signal for range profiles (averaging with $N=65$ range profiles) in the frequency domain, using the blanket method, as briefly described above.

The "Fractal Length" criterion examines the difference between the fractal length for the scale $\delta=1$ and the fractal length logarithm for the scale of $\delta$ (the signal for scale $\delta=1$ is essentially identical to the backscattered signal), see Fig. 8 and Fig. 9. Then the results for the grazing angles $10^{\circ}$ and $25^{\circ}$ are shown in Fig. 8 and Fig. 9.

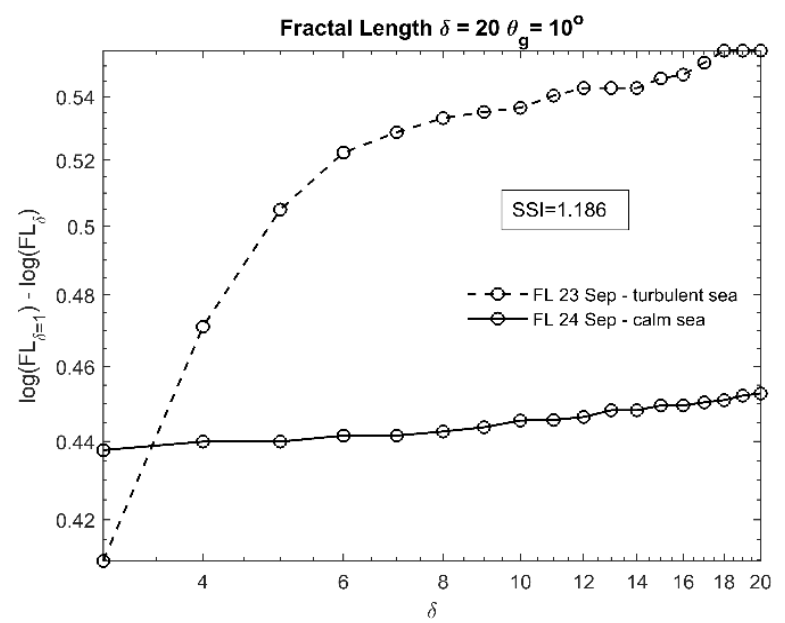

Fig. 8. Logarithmic Fractal Length as a function of iteration $\delta$ for grazing angle $10^{\circ}$.

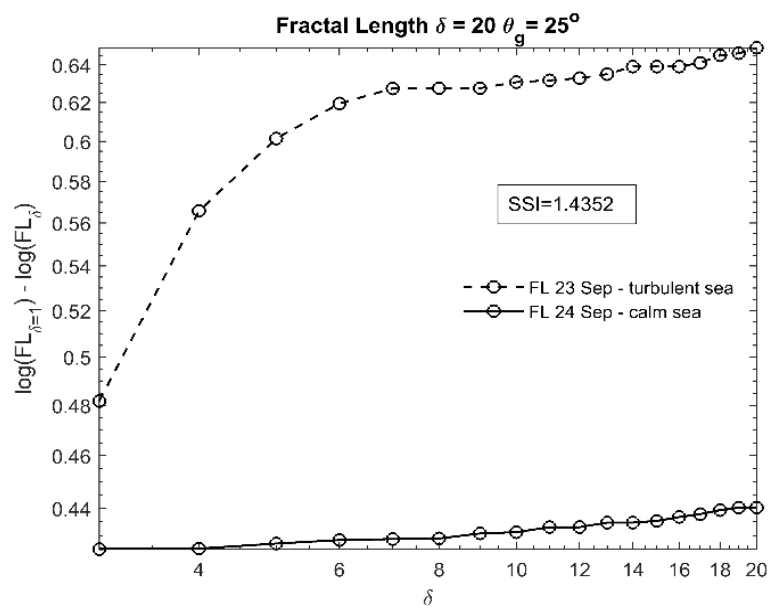

Fig. 9. Logarithmic Fractal Length as a function of iteration $\delta$ for grazing angle $25^{\circ}$.

Finally, the Sea State Index (SSI) is calculated for the fractal length criterion. The Sea State Index of the fractal 
length criterion is the ratio of the mean logarithmic fractal length in the case of turbulent sea to the mean logarithmic fractal length for calm sea, in the frequency domain. As shown in Table II, the values of SSI here are higher than 1 for all values of grazing angle (i.e., from $5^{\circ}$ to $35^{\circ}$ ), thus the fractal length criterion examined here is of high confidence.

TABLE II. SEA STATE INDEX (SSI) FOR FRACTAL LENGTH.

\begin{tabular}{|c|c|}
\hline Angle & SSI \\
\hline $5^{\circ}$ & 1.272 \\
\hline $10^{\circ}$ & 1.186 \\
\hline $15^{\circ}$ & 1.437 \\
\hline $20^{\circ}$ & 1.383 \\
\hline $25^{\circ}$ & 1.435 \\
\hline $30^{\circ}$ & 1.382 \\
\hline $35^{\circ}$ & 1.563 \\
\hline
\end{tabular}

\section{Variance $\sigma^{2}$ Criterion Results}

The "Variance $\sigma^{2}$ " criterion is another approach used, as already mentioned in Section III-B, (7).

Then, under this criterion, numerical calculations are performed for the variance $\sigma^{2}$ and the results are presented in Table III below (very satisfactory verification results).

TABLE III. VARIANCE RESULTS FOR TURBULENT AND CALM SEA AND RANGE PROFILES IN FREQUENCY DOMAIN.

\begin{tabular}{|c|c|c|}
\hline Angle & Turbulent & Calm \\
\hline $5^{\circ}$ & 0.856 & 0.279 \\
\hline $10^{\circ}$ & 0.606 & 0.328 \\
\hline $15^{\circ}$ & 1.121 & 0.085 \\
\hline $20^{\circ}$ & 1.070 & 0.300 \\
\hline $25^{\circ}$ & 0.915 & 0.229 \\
\hline $30^{\circ}$ & 0.720 & 0.243 \\
\hline
\end{tabular}

\section{Power Spectrum Density - Least Squares} Approximation Criterion Results

The "Power Spectrum Density - Least Squares" criterion is used here to validate the results of the two main fractal criteria. The least squares approximations of the power spectrum density results, as lines of the form $\alpha x+\beta$, are shown in Fig. 10 and Fig. 11 for the grazing angles of $10^{\circ}$ and $25^{\circ}$, respectively. Figure 10 and Figure 11 represent the power spectral density (PSD) versus the frequency on a loglog scale for turbulent and calm sea (see also [8], [9]).

In Fig. 10 and Fig. 11, it can be observed that the slopes of the lines of the least squares approximation (LSA) exhibit an absolute slope for the turbulent sea greater than the absolute slope value for the calm sea, something that is actually used as a criterion for characterizing the sea state. Then the corresponding numerical results are provided in Table IV.

TABLE IV. ABSOLUTE SLOPE OF LEAST SQUARES APPROXIMATION RESULTS OF POWER SPECTRUM DENSITY FOR TURBULENT AND CALM SEA.

\begin{tabular}{|c|c|c|}
\hline Angle & Turbulent & Calm \\
\hline $5^{\circ}$ & 0.290 & 0.119 \\
\hline $10^{\circ}$ & 0.368 & 0.146 \\
\hline $15^{\circ}$ & 0.316 & 0.166 \\
\hline $20^{\circ}$ & 0.300 & 0.148 \\
\hline $25^{\circ}$ & 0.332 & 0.117 \\
\hline $30^{\circ}$ & 0.346 & 0.115 \\
\hline $35^{\circ}$ & 0.314 & 0.174 \\
\hline
\end{tabular}

Concluding, in a manner similar to that described in [8], [9], the "Power Spectrum Density - Least Squares" criterion can be used to characterize the sea state and to verify the criteria of "Fractal Dimension" and "Fractal Length" in a satisfactory way.

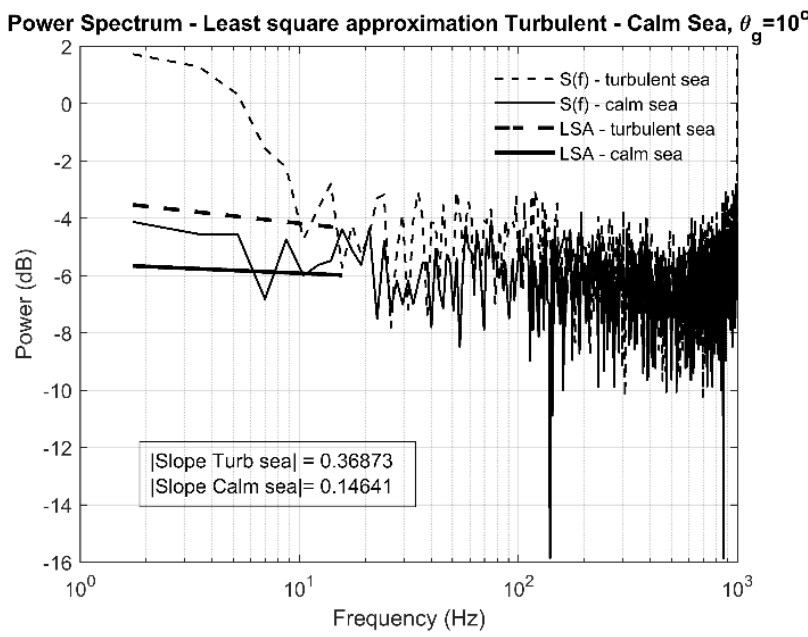

Fig. 10. Power Spectrum Density and Least Squares slope for grazing angle $10^{\circ}$.

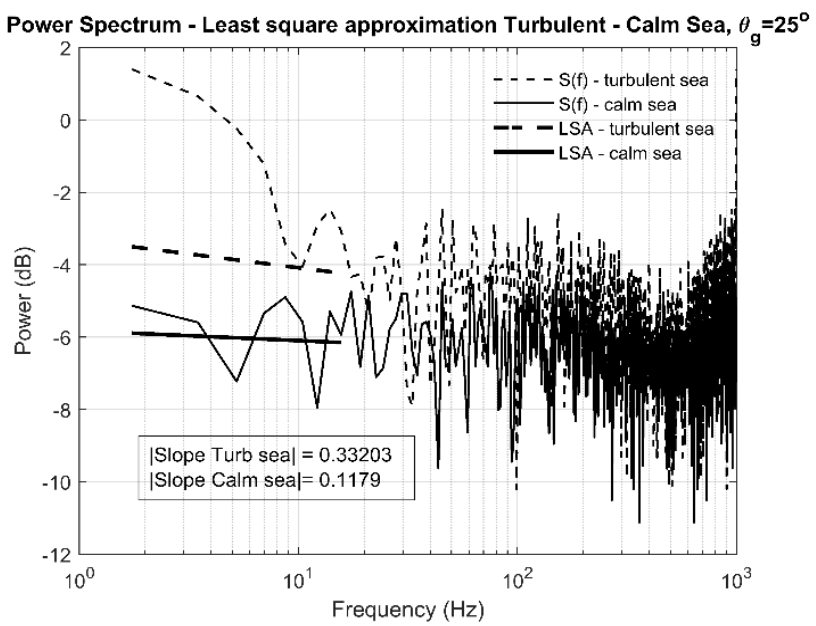

Fig. 11. Power Spectrum Density and Least Squares slope for grazing angle $25^{\circ}$.

\section{V.CONCLUSIONS AND FUTURE RESEARCH}

To summarize, this paper focused on the sea state characterization with the use of the four criteria mentioned above, where the two main criteria are based on the fractal theory ("Fractal Dimension" and "Fractal Length" criteria) and the other two criteria ("Variance $\sigma^{2 "}$ and "Power Spectrum Density - Least Squares" criteria) verify the two aforementioned fractal criteria.

The four criteria were applied to the experimental onedimensional signatures of a synthetic aperture radar (SAR) in the frequency domain (real radar data from sea surface) in two different sea states (turbulent and calm sea). The corresponding recorded sea clutter radar data were collected during the "NEMO 2014" trials in Taranto, Italy, 2324/9/2014. An X-band PicoSAR airborne radar was used for that purpose by FFI (i.e., "Norwegian Institute of Defense", Oslo, Norway).

The four criteria were applied to the backscattered radar signals from the sea surface. Namely, to suppress the inherent radar receiver electronic noise, averaging of the radar range profiles was used (here with $\mathrm{N}=65$ range profiles) and subsequently the data were normalized on a 
scale from 0 to 1 (i.e., "normalized range profiles"). Finally, the range profiles generated as described above, were transformed to the frequency domain. Lastly, the Sea State Index (SSI) was calculated for both the fractal dimension criterion and the fractal length criterion. All above criteria were found to be suitable for accurate sea state characterization.

In our future related research, we intend to validate the four criteria for sea state characterization presented above for simulated backscattered radar data (range profiles) to be produced by a rigorous electromagnetic (EM) code, already developed by our research group. Furthermore, we intend to use fractal methods on the "full set of range profiles" [i.e., three-dimensional (3D) fractal analysis on the backscattered radar range profiles], rather than the essentially twodimensional (2D) analysis of the range profiles, presented in this paper.

\section{ACKNOWLEDGMENT}

The authors would like to thank SET-215 Working Group, and in particular the FFI Institute (i.e., "Norwegian Institute of Defense", Oslo, Norway), for providing to us the real recorded sea clutter radar data, which were collected during the "NEMO 2014" trials in Taranto, Italy (see Fig. 2 above).

Furthermore, the authors acknowledge very useful discussions concerning the preparation of this paper, with Prof. N. Ampilova and Prof. I. Soloviev, Dept. of Information Science, Saint Petersburg State University, Russia, in the area of "fractal techniques".

\section{CONFLICTS OF INTEREST}

The authors declare that they have no conflicts of interest.

\section{REFERENCES}

[1] D. H. Werner and R. Mittra, "Fractal electrodynamics: Surfaces and superlattices", in Frontiers in Electromagnetics. Wiley-IEEE Press, 2000, pp. 1-47. DOI: 10.1109/9780470544686.ch1.

[2] K. Falconer, Fractal Geometry: Mathematical Foundations and Applications. J. Wiley and Sons, 1990. DOI: 10.2307/2532125.

[3] B. B. Mandelbrot, The Fractal Geometry of Nature. New York: W. H. Freeman and Company, 1977.

[4] B. B. Mandelbrot, Fractals: Form, Chance and Dimension. W. H. Freeman and Company, 1977.

[5] B. Mandelbrot, "How long is the coast of Britain? Statistical selfsimilarity and fractional dimension", Science, vol. 156 , no. 3775 , pp. 636-638, 1967. DOI: 10.1126/science.156.3775.636.

[6] R. Esteller, G. Vachtsevanos, J. Echauz, and B. Lilt, "Comparison of fractal dimension algorithms using synthetic and experimental data", in Proc. of ISCAS '99, IEEE International Symposium on Circuits and Systems, 1999. DOI: 10.1109/ISCAS.1999.778819.

[7] B. Li, Y. Xu, J. Zhang, and L. Cui, "Study on calculation models of curve fractal dimension", in Proc. of 6th International Conference on Natural Computation (ICNC 2010), 2010, pp. 3072-3079. DOI: 10.1109/ICNC.2010.5584690.

[8] T. Lo, H. Leung, J. Litva, and S. Haykin, "Fractal characterization of sea-scattered signals and detection of sea-surface targets", IEE Proceedings $F$ (Radar and Signal Processing), vol. 140, no. 4, pp. 243-250, 1993. DOI: 10.1049/ip-f-2.1993.0034.

[9] J. Hu, W.-W. Tung, and J. Gao, "Detection of low observable targets within sea clutter by structure function based multifractal analysis", IEEE Transactions on Antennas and Propagation, vol. 54, no. 1, pp. 136-143, 2006. DOI: 10.1109/TAP.2005.861541.

[10] J. Chen, T. K. Y. Lo, H. Leung, and J. Litva, "The use of fractals for modeling EM waves scattering from rough sea surface", IEEE Transactions on Geoscience and Remote Sensing, vol. 34, no. 4, pp. 966-972, 1996. DOI: 10.1109/36.508413.
[11] F. Berizzi, E. Dalle Mese, and G. Pinelli, "One-dimensional fractal model of the sea surface", IEE Proceedings - Radar, Sonar and Navigation, vol. 146, no. 1, pp. 55-64, 1999. DOI: 10.1049/iprsn:19990259.

[12] F. Berizzi and E. Dalle-Mese, "Fractal analysis of the signal scattered from the sea surface", IEEE Transactions on Antennas and Propagation, vol. 47, no. 2, pp. 324-338, 1999. DOI: 10.1109/8.761073.

[13] G. Lixin and Z. Wu, "Fractal model and electromagnetic scattering from time-varying sea surface", Electronics Letters, vol. 36, no. 21, pp. 1810-1812, 2000. DOI: 10.1049/el:20001199.

[14] Z. Li and Y.-Q. Jin, "Bistatic scattering from a fractal dynamic rough sea surface with a ship presence at low grazing-angle incidence using the GFBM/SAA", Microwave and Optical Technology Letters, vol. 31, no. 2, pp. 146-151, 2001. DOI: 10.1002/mop.1383.

[15] F. Berizzi and E. Dalle-Mese, "Scattering from a 2-D sea fractal surface: Fractal analysis of the scattered signal", IEEE Transactions on Antennas and Propagation, vol. 50, no. 7, pp. 912-925, 2002. DOI: 10.1109/TAP.2002.800695.

[16] C. Yang and H. Zhou, "The study of electromagnetic scattering by a target with fractal rough surface", in Proc. of 6th International Symposium on Antennas, Propagation and EM Theory, 2003, pp. 488-491. DOI: 10.1109/ISAPE.2003.1276734.

[17] Y. Li, X. Lv, K. Liu, and S. Zhao, "Fractal-based target detection within sea clutter", Acta Oceanol. Sin., vol. 33, no. 9, pp. 68-72, 2014. DOI: 10.1007/s13131-014-0519-1.

[18] A. Jayaprakash, G. Ramachandra Reddy, and N. S. S. R. K. Prasad, "Small target detection within sea clutter based on fractal analysis", Procedia Technology, vol. 24, pp. 988-995, 2016. DOI: 10.1016/j.protcy.2016.05.217.

[19] L. Gong et al., "Characterization and prediction of complex natural fractures in the tight conglomerate reservoirs: A fractal method", Energies, vol. 11, no. 9, p. 2311, 2018. DOI: 10.3390/en11092311.

[20] H. Pan et al., "Roughness change analysis of sea surface from visible images by fractals", IEEE Access, vol. 8, pp. 78519-78529, 2020. DOI: 10.1109/ACCESS.2020.2990161

[21] R. Sotner, O. Domansky, J. Jerabek, N. Herencsar, J. Petrzela, and D. Andriukaitis, "Integer-and Fractional-Order Integral and Derivative Two-Port Summations: Practical Design Considerations," Applied Sciences, vol. 10, no. 1, p. 54, Dec. 2019. DOI: 10.3390/app10010054.

[22] R. Sotner, J. Petrzela, J. Jerabek, N. Herencsar and D. Andriukaitis, "Design of Fractional-Order Integrator Controlled by Single Voltage Gain" in Proc. of 42nd International Conference on Telecommunications and Signal Processing (TSP), 2019, pp. 360364, doi: 10.1109/TSP.2019.8768814.

[23] S. Peleg, J. Naor, R. Hartley, and D. Avnir, "Multiple resolution texture analysis and classification", IEEE Transactions on Pattern Analysis and Machine Intelligence, vol. PAMI-6, no. 4, pp. 518-523, Jul. 1984. DOI: 10.1109/TPAMI.1984.4767557.

[24] A. Malamou, C. Pandis, P. Frangos, P. Stefaneas, A. Karakasiliotis, and D. Kodokostas, "Application of the modified fractal signature method for terrain classification from synthetic aperture radar images", Elektronika ir Elektrotechnika, vol. 20, no. 6, pp. 118-121, 2014. DOI: 10.5755/j01.eee.20.6.7281.

[25] Y. Y. Tang, H. Ma, D. Xi, X. Mao, and C. Y. Suen, "Modified fractal signature (MFS): A new approach to document analysis for automatic knowledge acquisition", IEEE Transactions on Knowledge and Data Engineering, vol. 9, no. 5, pp. 747-762, 1997. DOI: 10.1109/69.634753.

[26] S. Savaidis, P. Frangos, D. L. Jaggard, and K. Hizanidis, "Scattering from fractally corrugated surfaces using the extended boundary condition method", Journal of the Optical Society of America A, vol. 14, no. 2 , pp. $475-485$, 1997. DOI: 10.1364/JOSAA.14.000475.

[27] N. Lin, H. P. Lee, S. P. Lim, and K. S. Lee, "Wave scattering from fractal surfaces", Journal of Modern Optics, vol. 42, no. 1, pp. 225241, 1995. DOI: 10.1080/09500349514550181.

[28] D. Jaggard and X. Sun, "Scattering from fractally corrugated surfaces", Journal of the Optical Society of America A, vol. 7, no. 6, pp. 1131-1139, 1990. DOI: 10.1364/JOSAA.7.001131.

[29] A. K. Sultan-Salem and G. L. Tyler, "Validity of the Kirchhoff approximation for electromagnetic wave scattering from fractal surfaces", IEEE Transactions on Geoscience and Remote Sensing, vol. 42, no. 9, pp. 1860-1870, 2004. DOI: 10.1109/TGRS.2004.832655.

[30] R. Xin-Cheng and G. Li-Xin, "Fractal characteristics investigation on electromagnetic scattering from 2-D Weierstrass fractal dielectric rough surface", Chinese Physics B, vol. 17, no. 8, p. 2956, 2008. DOI: $10.1088 / 1674-1056 / 17 / 8 / 032$. 
[31] A. Iodice, A. Natale, and D. Riccio, "Kirchhoff scattering from fractal and classical rough surfaces: Physical interpretation", IEEE
Transactions on Antennas and Propagation, vol. 61, no. 4, pp. 21562163, 2013. DOI: 10.1109/TAP.2012.2236531.

This article is an open access article distributed under the terms and conditions of the Creative Commons Attribution 4.0 (CC BY 4.0) license (http://creativecommons.org/licenses/by/4.0/). 